\title{
Propuesta de un sistema de aprendizaje de lengua de señas basado en las tecnologías de la información y comunicación
}

\section{Proposal of a sign language learning system based on information and communication technologies}

\author{
Milton Alfredo Campoverde Molina ${ }^{1 *}$, Jacqueline Mishel Reina Alvarado ${ }^{1}$, Antonio Gabriel Carangui Delgado ${ }^{1}$ \\ Universidad Católica de Cuenca ${ }^{1}$ \\ *mcampoverde@ucacue.edu.ec
}

DOI: https://doi.org/10.26871/killkana_tecnica.v2i1.284

\begin{abstract}
Resumen
Esta investigación revela el desarrollo del software que con uso de dispositivos de reconocimiento de voz traduce a un lenguaje de señas; estableciendo como objeto de estudio a 10 estudiantes con discapacidad auditiva de la Unidad Educativa Especial Claudio Neira Garzón: Segundo de Básica (1 estudiante), Cuarto de Básica (5 estudiantes) y Quinto de Básica (4 estudiantes), a continuación, se realizó un análisis del porcentaje de discapacidad de cada estudiante de acuerdo al carnet del Consejo Nacional para la Igualdad de Discapacidades (CONADIS), luego se aplicó una encuesta a los estudiantes y se mantuvo una entrevista con la directora y subdirectora de la Unidad Educativa, determinando que sería de gran apoyo utilizar alternativas tecnológicas en el proceso de enseñanza-aprendizaje de la lengua de señas en los niños de 7 y 15 años con déficit auditivo parcial o total dentro de la asignatura de Lengua y Literatura en la lectoescritura, para el desarrollo del software se utilizó la metodología en cascada, luego de haber desarrollado el sistema se hicieron varias pruebas obteniendo muy buenos resultados en cuanto a su uso, incrementando el nivel de compresión y entendimiento del lenguaje español y la lengua de señas en los niños y además hubo una excelente acogida por parte de los docentes y estudiantes que demostraron mucho interés por usar el software en el proceso de enseñanza-aprendizaje.
\end{abstract}

Palabras clave: Discapacidad auditiva, educación, lenguaje señas, software, TIC.

\begin{abstract}
This research reveals the development of software that translates into sign language with the use of speech recognition devices; establishing as object of study 10 students with hearing disability of the Claudio Neira Garzón Special Educational Unit: Basic Second (1 student), Basic Room (5 students) and Basic Fifth (4 students), followed by a analysis of the percentage of disability of each student according to the card of the National Council for the Equality of Disabilities (CONADIS), then a survey was applied to the students and an interview was held with the director and assistant director of the Educational Unit, determining what would be of great support to use technological alternatives in the teachinglearning process of sign language in children of 7 and 15 years with partial or total auditory deficit within the subject of Language and Literature in reading and writing, for the development of software He used the methodology in cascade, after having developed the system, several tests were made obtaining very good results. $n$ in terms of its use, increasing the level of understanding and understanding of the Spanish language and sign language in children and in addition there was an excellent reception from teachers and students who showed great interest in using the software in the teaching process -learning.
\end{abstract}

Key words: Hearing impairment, education, sign language, software, TIC.

\section{INTRODUCCIÓN}

La Constitución de la República del Ecuador 2008 [1] en el artículo 26 considera que "la educación es un derecho de las personas a lo largo de su vida y un deber ineludible e inexcusable del estado. Del mismo modo, en el artículo 46, numeral 3 estipula la "atención preferente para la plena integración social de quienes tengan discapacidad. El estado garantizará su incorporación en el sistema de educación regular y en la sociedad". También, reconoce a las personas con discapacidad, los derechos a: (7.-) Una educación que desarrolle sus potencialidades y habilidades para su integración y participación en igualdad de condiciones. Se garantizará su educación dentro de la educación regular. Los establecimientos educativos cumplirán normas de accesibilidad para personas con discapacidad e implementarán un sistema de becas que responda a las 
condiciones económicas de este grupo.

De la misma forma, en el Plan Nacional de Desarrollo 2017-2021. Toda una Vida [2] se plantea como primer objetivo "Garantizar una vida digna con iguales oportunidades para todas las personas", estableciendo como una de sus Políticas numeral 1.4. "Garantizar el desarrollo infantil integral para estimular las capacidades de los niños y niñas, considerando los contextos territoriales, la interculturalidad, el género y las discapacidades" y como una de sus metas "dotar de ayudas técnicas a las personas con discapacidad a 2021”; asimismo, el CONADIS [3] en sus normas jurídicas en discapacidad Ecuador establece:

- “Acceso a una educación inclusiva y especializada de calidad a las personas con discapacidad que atienda sus necesidades específicas" (educación), así como el "Acceso al medio físico, Servicios de transporte, Tecnologías de la información y comunicación".

- Dotar o repotenciar la infraestructura, el equipamiento, la conectividad y el uso de TIC, recursos educativos y mobiliarios de los establecimientos de educación pública, bajo estándares de calidad, adaptabilidad y accesibilidad, según corresponda. (4.1.c.-PNBV).

- Una educación que desarrolle sus potencialidades y habilidades para su integración y participación en igualdad de condiciones. Se garantiza su educación dentro de la educación regular. Los planteles regulares incorporarán trato diferenciado y los de atención especial la educación especializada. Los establecimientos educativos cumplirán normas de accesibilidad para personas con discapacidad e implementarán un sistema de becas que responda a las condiciones económicas de este grupo.

La OMS en su informe mundial sobre la discapacidad 2011 estima que más de mil millones de personas viven con algún tipo de discapacidad; o sea, alrededor del $15 \%$ de la población mundial (según las estimaciones de la población mundial en 2010). Esta cifra es superior a las estimaciones previas de la Organización Mundial de la Salud, correspondientes a los años 1970, que eran de aproximadamente un $10 \%$ [4].

Según, las estadísticas publicadas por el Consejo Nacional para la Igualdad de Discapacidades (CONADIS) [5] con información del Ministerio de Salud Pública de las personas registradas con discapacidad en el Ecuador, Azuay y Cuenca se puede ver la tendencia de crecimiento de las discapacidades: auditiva, física, intelectual, lenguaje, psicosocial y visual [6] [7] [8] en el tiempo en las Fig. 1, 2, 3 respectivamente:
Fig. 1. Tendencia de la discapacidad en Ecuador.

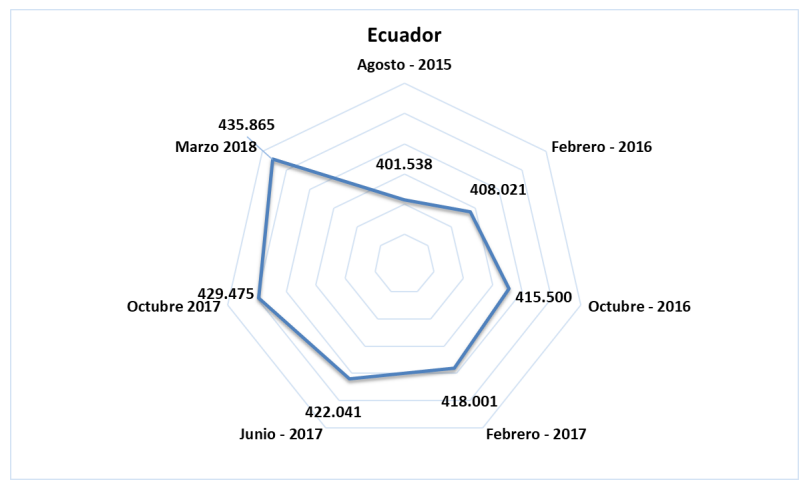

Fig. 2. Tendencia de la discapacidad en el Azuay.

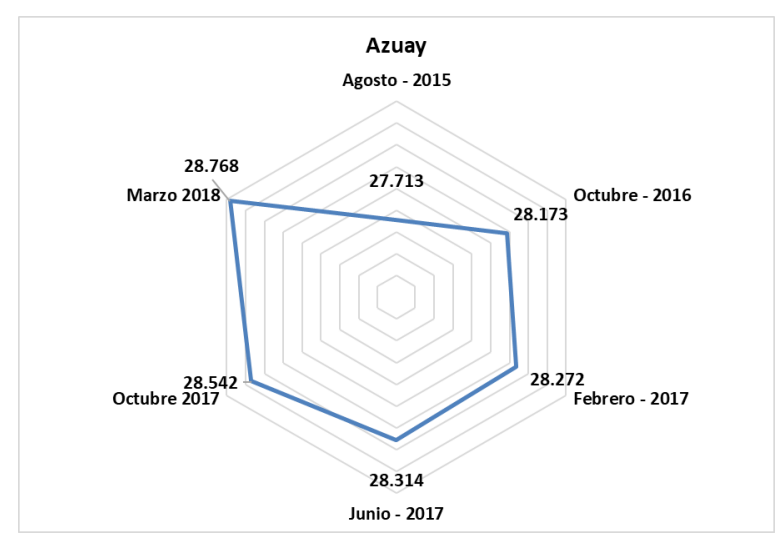

Fig. 3. Tendencia de la discapacidad en el Cuenca.

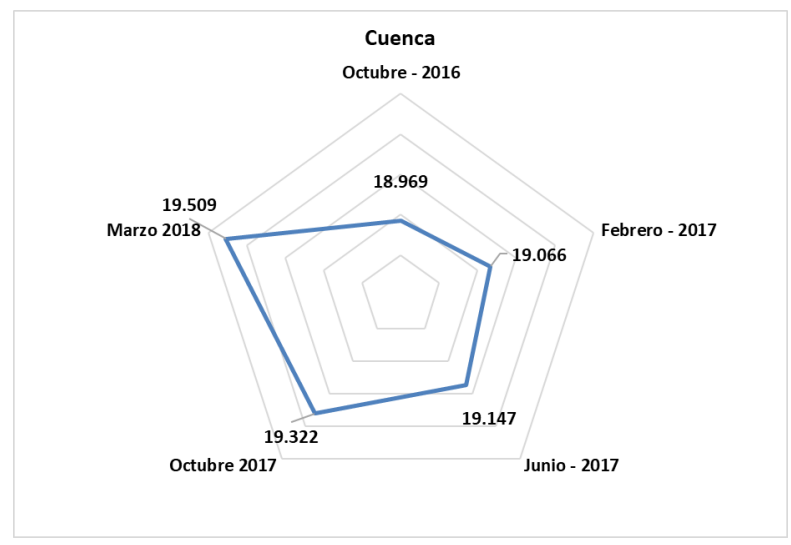

De la misma manera, en base a las estadísticas publicadas por el Consejo Nacional para la Igualdad de Discapacidades (CONADIS) [5] con información del Ministerio de Salud Pública de las personas registradas con discapacidad en el Ecuador, Azuay y Cuenca se puede ver la tendencia de crecimiento de la discapacidad auditiva [6] [7] [8] en el tiempo en las Fig. 4, 5, 6 respectivamente: 
Fig. 4. Tendencia de la discapacidad auditiva en el Ecuador.

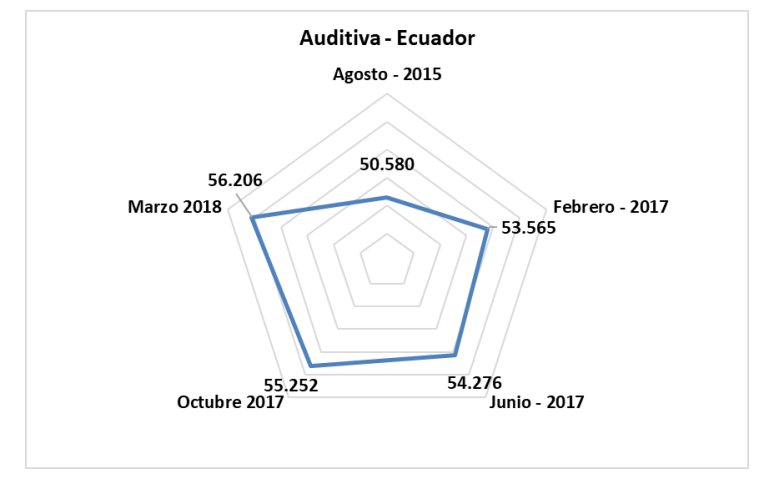

Fig. 5. Tendencia de la discapacidad visual en el Ecuador.

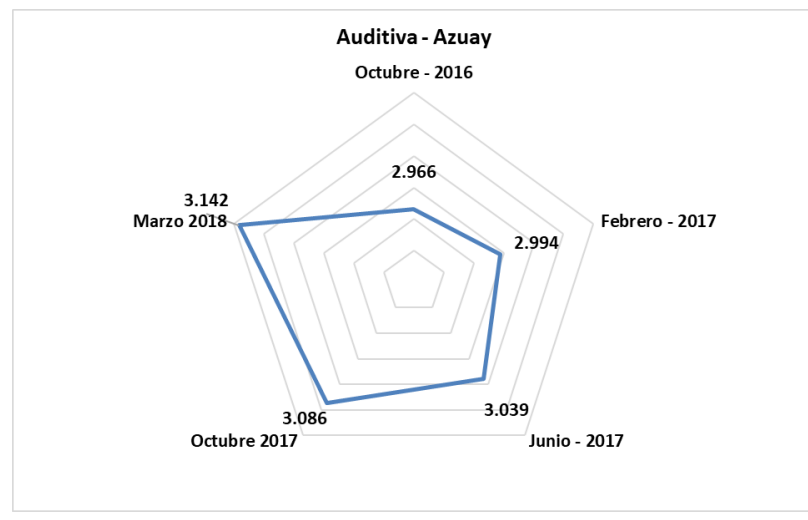

Fig. 6. Tendencia de la discapacidad auditiva Cuenca - Ecuador.

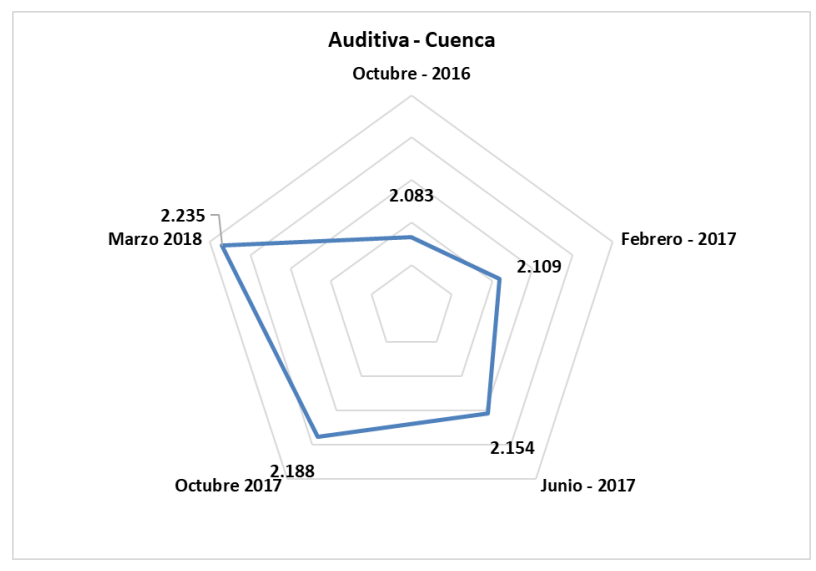

Haciendo, un análisis de los gráficos expuestos anteriormente se puede ver el crecimiento en número de personas con discapacidad en el tiempo en el Ecuador, Azuay y Cuenca; teniendo en cuenta, que las causas [9] de discapacidades en el Ecuador han sido originadas por: accidente de trabajo, accidente de tránsito, accidente deportivo, accidente doméstico, congénito/genético, desastres naturales/guerra, enfermedad adquirida, problemas de parto, trastornos nutricionales, violencia y otros (no se sabe), razón por lo cuál esta investigación busca alternativas tecnológicas que fomenten un aporte significativo en la enseñanza-aprendizaje de niños con discapacidad auditiva. Contemplando, que en el ámbito educativo las TIC son consideradas valiosas herramientas de trabajo que pueden contribuir al desarrollo personal con un carácter individualizado e independiente pero integracionista y creativo. Por otra parte, su utilización en las distintas actividades económicas, sociales, culturales, entre otras, ha impuesto la necesidad de que la escuela asuma un rol activo en la preparación del futuro ciudadano para la utilización de dichas tecnologías, de manera que pueda utilizarlas para su desarrollo [10]. Si se quiere, lograr una educación inclusiva [11], es necesario determinar cómo deben ocupar ese espacio las personas sordas. En primer lugar hay que establecer que el problema más grande que enfrentan estos estudiantes es un problema de comunicación. Sin la posibilidad de comunicarse y acceder a la información que se maneja en las escuelas, es difícil, si no imposible acceder a "una educación con equidad", si no hay un idioma común con el cual se pueda comunicar con su maestro o maestra.

Considerando todos los puntos tratados en los párrafos anteriores, esta investigación plantea como objetivo desarrollar un software que con uso de dispositivos de reconocimiento de voz traduzca a lenguaje de señas y sirva como herramienta de trabajo en la labor educativa de la Unidad Educativa Especial Claudio Neira Garzón en beneficio de sus niños con discapacidad auditiva.

\section{MARCO TEÓRICO}

\section{A. Discapacidad Auditiva}

La Ley Orgánica de Discapacidades del Ecuador [12] en el artículo 6 establece que "se considera persona con discapacidad a toda aquella que, como consecuencia de una o más deficiencias físicas, mentales, intelectuales o sensoriales, con independencia de la causa que la hubiera originado, ve restringida permanentemente su capacidad biológica, sicológica y asociativa para ejercer una o más actividades esenciales de la vida diaria"; también, en el artículo 33 se revela que la autoridad educativa nacional en el marco de su competencia, vigilará y supervisará, en coordinación con los gobiernos autónomos descentralizados, que las instituciones educativas escolarizadas y no escolarizadas, especial y de educación superior, públicas y privadas, cuenten con infraestructura, diseño universal, adaptaciones físicas, ayudas técnicas y tecnológicas para las personas con discapacidad, adaptación curricular, participación permanente de guías intérpretes, según la necesidad y otras medidas de apoyo personalizadas y efectivas que fomenten el desarrollo académico y social de las personas con discapacidad.

La discapacidad auditiva se define como la dificultad que presentan algunas personas para participar en actividades propias de la vida cotidiana, que surge como consecuencia de la interacción entre una dificultad específica para percibir a través de la audición los sonidos del ambiente y dependiendo del grado de pérdida auditiva, los sonidos 
del lenguaje oral, y las barreras presentes en el contexto en el que se desenvuelve la persona.Tener una dificultad auditiva no implica tener restricciones para comunicarse. Una cosa es el proceso de comunicación y otra el medio y el canal empleados para hacerlo. Lo que una persona con dificultades auditivas puede tener son restricciones para la utilización del lenguaje oral como medio exclusivo de comunicación, sin embargo, las posibilidades de comunicar mediante otros medios están intactas. Por este motivo, las personas sordas desarrollan medios diferentes para comunicarse, como la lengua de señas [13].

\section{B. Lengua de Señas}

Hoy en día los niños con discapacidad auditiva [14] presentan grandes dificultades para comunicarse, ya que su principal lenguaje de comunicación es el lenguaje de señas, pero es necesario enseñar a los niños a comunicarse a través de la lectura y la escritura para incorporarse dentro de la sociedad.

La Ley Orgánica de Discapacidades del Ecuador [12] en el artículo 70 reconoce la lengua de señas ecuatoriana como lengua propia y medio de comunicación de las personas con discapacidad auditiva. Se incorporará progresivamente el servicio de intérpretes de la lengua de señas ecuatoriana en las instituciones públicas, así como la capacitación de las y los servidores públicos en la misma. La lengua de señas [15] del país es conocida actualmente como Lengua de Señas Ecuatoriana, LSE. En los textos escritos sobre esta lengua en inglés se la representa con las siglas LSEC.

El lenguaje de señas constituye el principal recurso para la comunicación dentro de la comunidad sorda, que la aprende de forma natural y la convierte en una lengua necesaria, útil y práctica[16].

\section{Discapacidad Sensorial}

Los alumnos y alumnas con discapacidad sensorial son aquellos que presentan una deficiencia en su audición o visión con resultado de una percepción y procesamiento de la información disfuncionales, dificultándoles aspectos cognitivos en la adquisición y manipulación del espacio, en el lenguaje, en la relación personal y de funcionamiento en la comunidad. Por ello, y para que su desarrollo personal evolucione en el sentido general o de la normalidad poblacional, precisarán de los apoyos y recursos que compensen sus dificultades y refuercen sus habilidades. Dada la importancia que tienen los canales auditivo y visual en la adquisición de la información, se pasará a revisar sus dos discapacidades [17].

\section{Lectoescritura}

A lo largo de la historia, la concepción y status social de la lectoescritura ha evolucionado pasando de ser un "conocimiento" de los más privilegiados, a un aprendizaje fundamental para la integración en la sociedad actual, en la cual, autonomía y eficacia son dos condiciones esenciales [18]. La escalera de ubicación propuesta por Grossi, indica los conocimientos y habilidades en cada nivel, en los cuales ascender es sinónimo de aprendizaje y evolución en el proceso cognitivo [19], así:

- Nivel Presilábico 1: El estudiante expresa su conocimiento a través de dibujos y/o grafismos no convencionales.

- Nivel Presilábico 2: El estudiante emplea códigos similares con regularidad para expresar las mismas ideas, empieza a establecer códigos definidos.

- Nivel silábico: El estudiante tiene en cuenta el número de sílabas de la palabra y de acuerdo a ello coloca la cantidad de símbolos.

- Nivel Alfabético: El estudiante emplea códigos reconocidos y aceptados para expresarse pero comete adiciones $\mathrm{u}$ omisiones dentro de algunas palabras, en este proceso comienza a perfeccionar su nivel escritor.

- Nivel Alfabetizado: En este nivel los estudiantes escriben y reconocen los códigos establecidos para la comunicación en su lengua y es capaz de producir textos ya sea bajo orientación o por su propia iniciativa. Es en este nivel donde se determina que el estudiante ha logrado un aprendizaje y dominio del proceso lectoescritor.

El desarrollo de la lectoescritura implica los siguientes pasos en el proceso de la conciencia cognitiva: primero, pasar de la no-conciencia de la relación entre la escritura y el lenguaje hablado; a asociar lo escrito con el lenguaje oral; y al dominio de los signos escritos referidos directamente a objetos o entidades. Segundo, pasar del proceso de operaciones conscientes como la individualización de los fonemas, la representación de estos fonemas en letras, la síntesis de las letras en la palabra, la organización de las palabras; a la automatización de estas operaciones; y al dominio del texto escrito y del lenguaje escrito. El lenguaje escrito es una forma compleja de actividad analítica, en la cual la tarea fundamental es la toma de conciencia de la construcción lógica de la idea [20].

\section{E. Las Tecnologías de la Información y la Comunicación en la educación}

La educación se encuentra en un proceso de transformación de los procesos de enseñanza-aprendizaje para adaptarse a los cambios tecnológicos de la sociedad del siglo XXI, por la motivación de docentes e investigadores que han visto en las tecnologías un motor de cambio del sistema educativo y del quehacer de sus agentes [21].

Por tal razón, los currículos de la Educación General Básica (EGB) y el Bachillerato General Unificado (BGU) [22] que se implementan mediante Acuerdo Nro. MINEDUCME-2016-00020-A del 17 de febrero de 2016 en el Ecuador, tienen como prioridad el uso habitual de las tecnologías de la información y de la comunicación como instrumento facilitador para el desarrollo del currículo en cada una de las áreas. Para el efecto, de acuerdo a un análisis pedagógico y curricular, este proceso puede impulsarse desde los primeros años escolares y fortalecerse en la Básica Superior 
y Bachillerato, con la finalidad de que los estudiantes desarrollen destrezas suficientes que le faciliten el uso de los programas básicos de ofimática, como herramientas tecnológicas y didácticas, para su aprendizaje, además de fomentar el conocimiento en las Ciencias de la Computación. Con esta finalidad, dependiendo de la disponibilidad de cada una de las instituciones, los docentes de todas las áreas tanto del nivel de Básica como del Bachillerato, deberán planificar y desarrollar sus clases de las diferentes asignaturas utilizando los laboratorios de informática, es necesario organizar un horario de uso de laboratorios, garantizando su máxima capacidad durante la jornada escolar. Además, de sus horas pedagógicas, el docente de informática deberá programar capacitaciones para los docentes de las demás especialidades que garanticen su actualización digital.

\section{F. La discapacidad auditiva en el Ecuador}

Según, los antecedentes de personas con discapacidad auditiva en la educación [15]:

- 1940: Enriqueta Santillán inicia actividades docentes con niños sordos en Quito.

- 1952: Se funda el Instituto Mariana de Jesús, por iniciativa privada. El instituto atendía niños con discapacidad visual y con discapacidad auditiva.

- 1962: se funda el Instituto Nacional de Audición y Lenguaje (INAL), en Quito, a partir de lo que fue una sección para niños con necesidades especiales en el Colegio Espejo.

- 1966: Varios jóvenes sordos comienzan a agruparse en Quito para organizar eventos deportivos.

- 1975: Se crea la Sociedad de Sordos Adultos Fray Luis Ponce de León, en Quito.

- 1976: Una sociedad similar a la quiteña se crea en Guayaquil.

- 1982: Se inicia el Proyecto "Mano a Mano" (estudio de la lengua de señas local) en la Sociedad Ponce de León.

- 1984: Se crea la asociación de sordos de Guayas.

- 1986: Se funda la Federación Nacional de Sordos de Ecuador.

- 1988: Aparece como libro una compilación de señas usadas por los sordos de Ecuador, resultado del Proyecto "Mano a Mano".

- 2012: Se publican el Diccionario Oficial de Lengua de Señas Ecuatoriana y un estudio sociolingüístico sobre la LSE (SIL International).

\section{G. Inclusión Educativa}

La educación inclusiva es el proceso que ofrece a todos los niños y niñas, sin distinción de discapacidad, raza o cualquier otra diferencia, la oportunidad para continuar siendo miembro de la clase ordinaria y para aprender de sus compañeros, juntamente con ellos, dentro del aula [23].

La UNESCO define la educación inclusiva como el proceso de identificar y responder a la diversidad de las necesidades de todos los estudiantes a través de la mayor participación en el aprendizaje, las culturas y las comunidades, y reduciendo la exclusión en la educación. Involucra cambios y modificaciones en contenidos, aproximaciones, estructuras y estrategias, con una visión común que incluye a todos los niño/as del rango de edad apropiado y la convicción de que es la responsabilidad del sistema regular, educar a todos los niño/as [24].

Hacer efectivo el derecho a la educación exige garantizar que todos los niños, niñas y jóvenes tengan, en primer lugar, acceso a la educación, pero no a cualquier educación sino a una de calidad con igualdad de oportunidades. Son, justamente, esos tres elementos los que definen la inclusión educativa o educación inclusiva. Podríamos decir entonces, que la inclusión educativa es consustancial al derecho a la educación o, en forma más definitoria, un requisito del derecho a la educación, toda vez que el pleno ejercicio de este derecho, implica la superación de toda forma de discriminación y exclusión educativa[25].

La inclusión educativa presenta una complejidad que puede ser comprendida de mejor manera si se tiene atención sobre el profesor como agente relevante y clave de este proceso. Puede constituirse en una barrera o en un agente facilitador de las prácticas inclusivas. Las actitudes del profesor acerca de la inclusión educativa, entendidas como el conjunto de percepciones, creencias, sentimientos y formas de actuar, impactan la disposición hacia la inclusión de personas con NEE. Una actitud positiva hacia prácticas inclusivas va a favorecer dicho proceso. En tanto una actitud negativa minimizará las oportunidades de aprendizaje y participación de estudiantes con algún tipo especial de necesidades educativas [26].

\section{Metodología}

La metodología utilizada en el desarrollo del software de aprendizaje de la lengua de señas mediante reconocimiento de voz es la metodología en cascada que consta de las siguientes fases [27]:

1) Definición de los requisitos: los servicios, restricciones y objetivos son establecidos con los usuarios del sistema. Se busca hacer esta definición en detalle.

2) Diseño de software: se divide el material educativo computarizado en sistemas de software o hardware. Se establece la arquitectura total del material. Se identifican y describen las abstracciones y relaciones de los componentes del sistema.

3) Implementación y pruebas unitarias: construcción de los módulos y unidades del material educativo computarizado. Se realizan pruebas de cada unidad.

4) Integración y pruebas del sistema: se integran todas las unidades, se prueban en conjunto, se entrega y aplica el conjunto probado al cliente.

5) Operación y mantenimiento: generalmente es la fase más extensa. El material educativo computarizado es puesto en marcha y se realiza la corrección de errores descubiertos. Se realizan mejoras de implementación. Se identifican nuevos requisitos. 
La interacción entre fases puede observarse en la Fig. 7. Cada fase tiene como resultado documentos que deben ser aprobados por el usuario:

Fig. 7. Modelo de desarrollo en cascada [27].

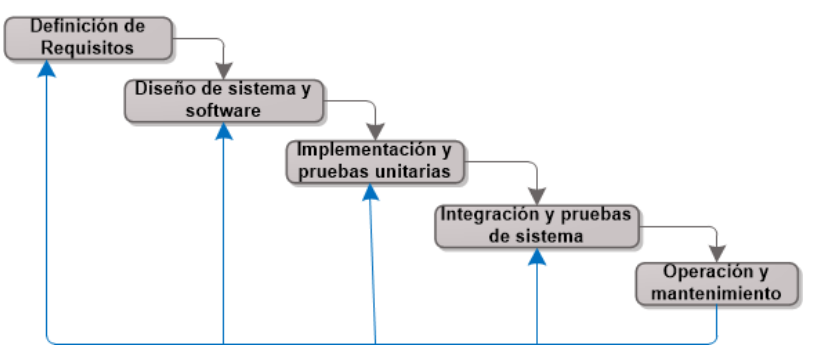

\section{RESUltados}

\section{A. Definición de los requisitos}

Esta investigación forma parte del proyecto de investigación científica "La accesibilidad tecnológica como estrategia de inclusión educativa", ejecutado por docentes investigadores y estudiantes de la carrera de Ingeniería de Sistemas de la Universidad Católica de Cuenca; la misma que se está desarrollando en la Unidad Educativa Especial Claudio Neira Garzón. Según su reseña histórica se puede ver que en el año de 1971 se crea con el nombre de Instituto Fiscal Especial de Invidentes y Sordos del Azuay, con Acuerdo Ministerial No.16, dictado por la Dirección Provincial de Educación, desde entonces han tomado en cuenta la necesidad social y humana de dar oportunidad de educación y cultura a las personas y niños con capacidades especiales físicas o intelectuales que les impidan desarrollarse y a su vez ser útiles en la sociedad. En el año 2014 se dio el cambio de denominación del establecimiento, según la normativa legal vigente como Unidad Educativa Especial Claudio Neira Garzón, nombre que representa al fundador Dr. Claudio Neira Garzón, profesor de invidentes desde enero de 1972 hasta diciembre de 1980 de la Unidad Educativa. En la actualidad, se cuenta con 69 estudiantes 40 con discapacidad auditiva y 29 con discapacidad visual, desde estimulación temprana hasta décimo año de educación básica. En base a una entrevista con la Directora y Subdirectora de la Unidad Educativa, se toma como población a los 40 estudiantes con discapacidad auditiva, determinando que sería de gran apoyo utilizar alternativas tecnológicas en el proceso de enseñanza-aprendizaje de la lengua de señas en los niños de 7 a 15 años. La área de estudio con la cual se va a trabajar es la asignatura de Lengua y Literatura dentro de la lectoescritura, teniendo en cuenta, que las señas, gestos e imágenes, es el principal modo de comunicación entre las personas con discapacidad auditiva, razón por la cual, esta investigación establece como objeto de estudio a los 10 estudiantes de: Segundo de Básica (1 estudiante), Cuarto de Básica (5 estudiantes) y Quinto de Básica (4 estudiantes) para el desarrollo del software que con uso de dispositivos de reconocimiento de voz traduzca a lenguaje de señas.
Luego de la determinación del objeto de estudio se realizó un diagnóstico del porcentaje de discapacidad de los 10 estudiantes según su diagnóstico clínico del carnet del CONADIS como se puede ver en la Fig. 8.

Fig. 8. Porcentaje de discapacidad auditiva de cada estudiante.

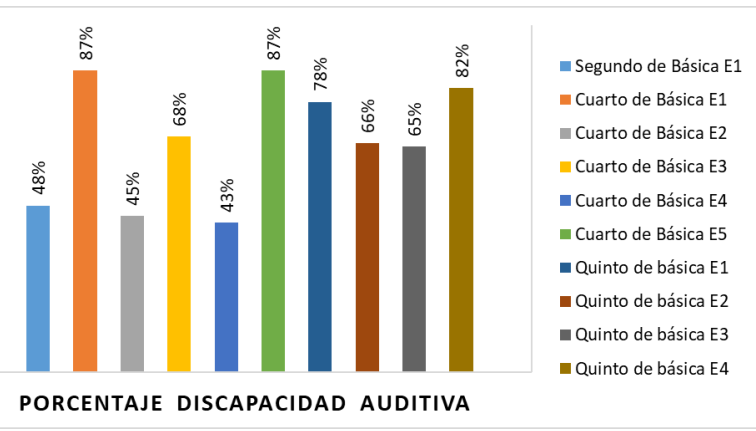

Asimismo, se realizaron 7 interrogantes para determinar las necesidades instruccionales que tienen los niños al momento de la enseñanza-aprendizaje del lenguaje de señas como se puede ver en la Fig. 9:

Fig. 9. Resultados de las interrogantes realizadas a los docentes.

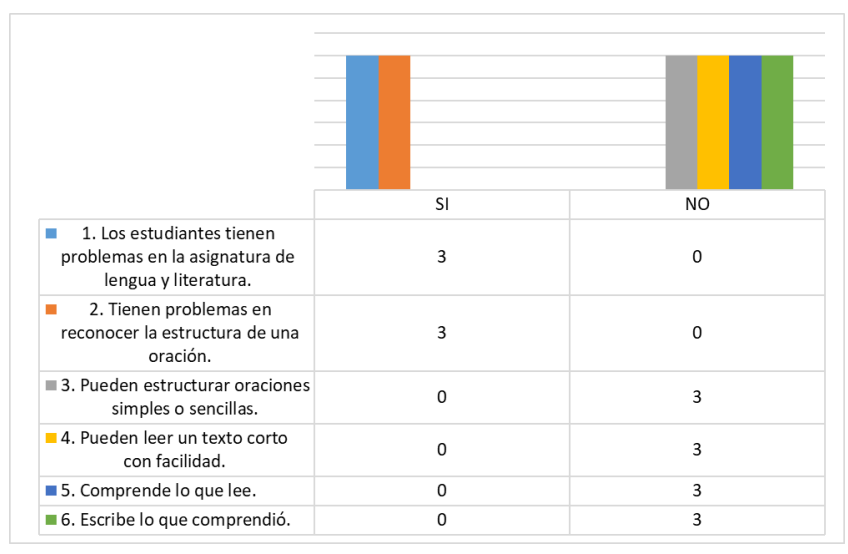

Con referencia a las Fig. 9 se evidencia que todas las preguntas realizadas a los docentes del Segundo, Cuarto y Quinto de Básica obtienen una puntuación de 3, que es el $100 \%$ en cada pregunta. Además, los docentes supieron manifestar que:

- La escritura y lectura lo hacen con ayuda y luego de que ha sido explicado varias veces en lengua de señas.

- Falta de conocimiento del lenguaje de señas.

- No hay lectura comprensiva.

- No escriben correctamente con las reglas gramaticales.

- No hay memoria de largo plazo.

- En la lengua de señas la estructura es diferente de la gramática española.

\section{Requerimientos Administrador}

1) R1. Realizar el mantenimiento de los registros del Sistema, a través del ingreso de la información general de 
los estudiantes, categorías, subcategorías, vocabulario, diccionario, oraciones simples y juegos, ver Fig. 10.

\section{Requerimientos Profesor}

1) R2. Registrar la planificación de las actividades por cada estudiante para un periodo de tiempo establecido, ver Fig. 11.

2) R3. Evaluar el aprendizaje alcanzado por cada estudiante a través del reporte de estudiantes para su retroalimentación, ver Fig. 11.

\section{Requerimientos Estudiante}

1) R5. Realizar la ejecución de las actividades planificadas por parte de los docentes, ver Fig. 12.

\section{Diagramas de Casos de Uso}

Un Diagrama de Caso de Uso muestra la relación entre los actores y los casos de uso del sistema. Representa la funcionalidad que ofrece el sistema en lo que se refiere a su interacción externa [28].

Fig. 10. Diagrama de Caso de Uso del Administrador del Sistema.

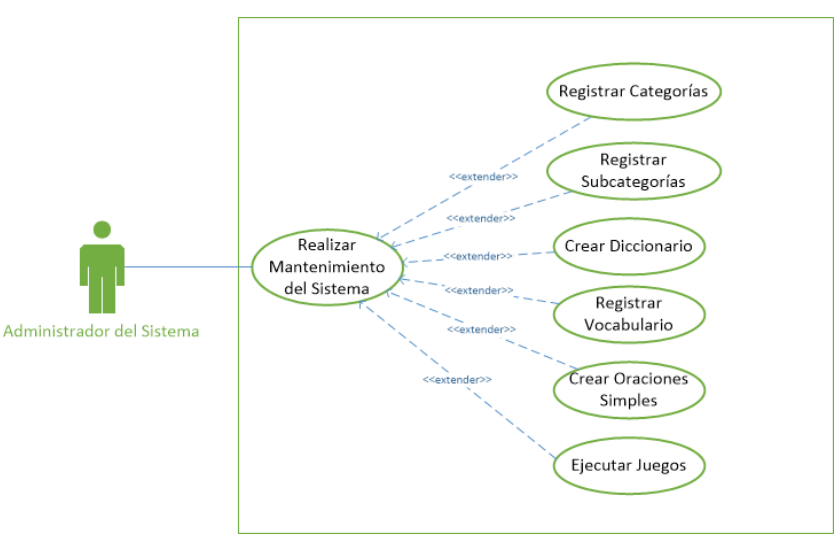

Fig. 11. Diagrama de Caso de Uso de los Profesores.

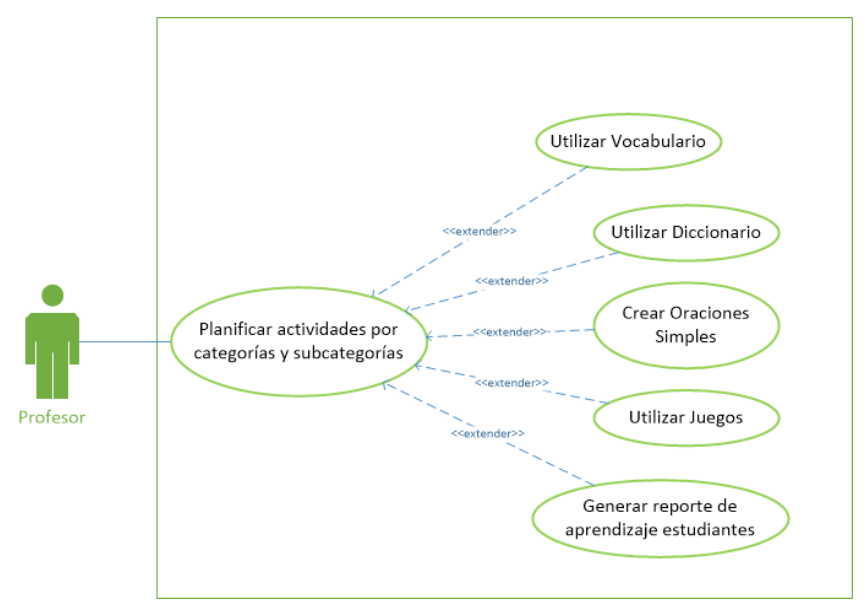

Fig. 12. Diagrama de Caso de Uso de los Estudiantes.

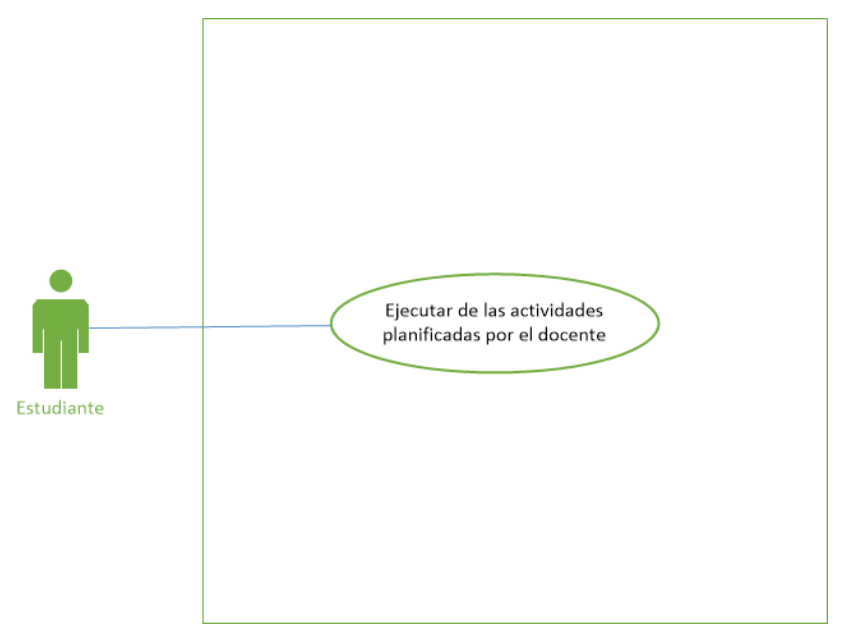

Los diagramas de casos de uso permiten visualizar la relación de cada actor con los procesos del sistema.

\section{B. Diseño de software}

\section{Arquitectura}

La arquitectura del software para el aprendizaje de la lengua de señas se puede ver en la Fig. 13, el mismo que esta diseñado en tres capas:

Fig. 13. Diagrama de Bloques de la Arquitectura del Software de aprendizaje de la lengua de señas.

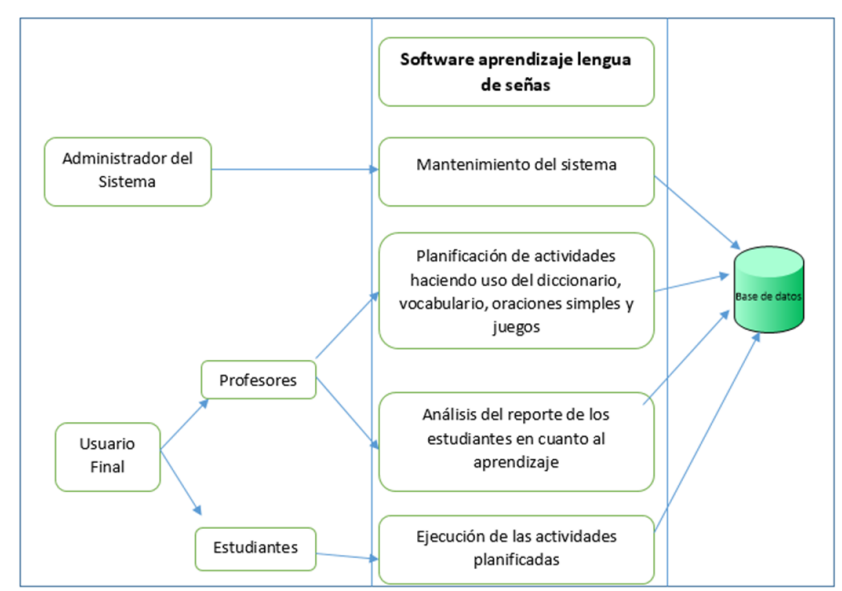

- Capa de Presentación: Se encarga de que el sistema interactúe con el usuario y viceversa: interfaces de administración del sistema, profesor y estudiante.

- Capa de Negocio: Permite la funcionalidad del sistema a través del mantenimiento del sistema, planificación de actividades haciendo uso del diccionario, vocabulario, oraciones simples y juegos, análisis de los reportes de los estudiantes en cuanto al aprendizaje y ejecución de las actividades programadas.

- Capa de Datos: Esta capa permite el almacenamiento de los datos del sistema y de los usuarios en una base de datos local. 


\section{Modelo entidad relación}

El modelo de datos de entidad-relación (ER) se basa en una percepción de un mundo real que consiste en un conjunto de objetos llamados entidades y las relaciones entre estos objetos. A continuación, en la Fig. 14 se puede observar el modelo entidad relación del Software de aprendizaje de la lengua de señas:

Fig. 14. Modelo entidad relación del Software de aprendizaje de la lengua de señas.

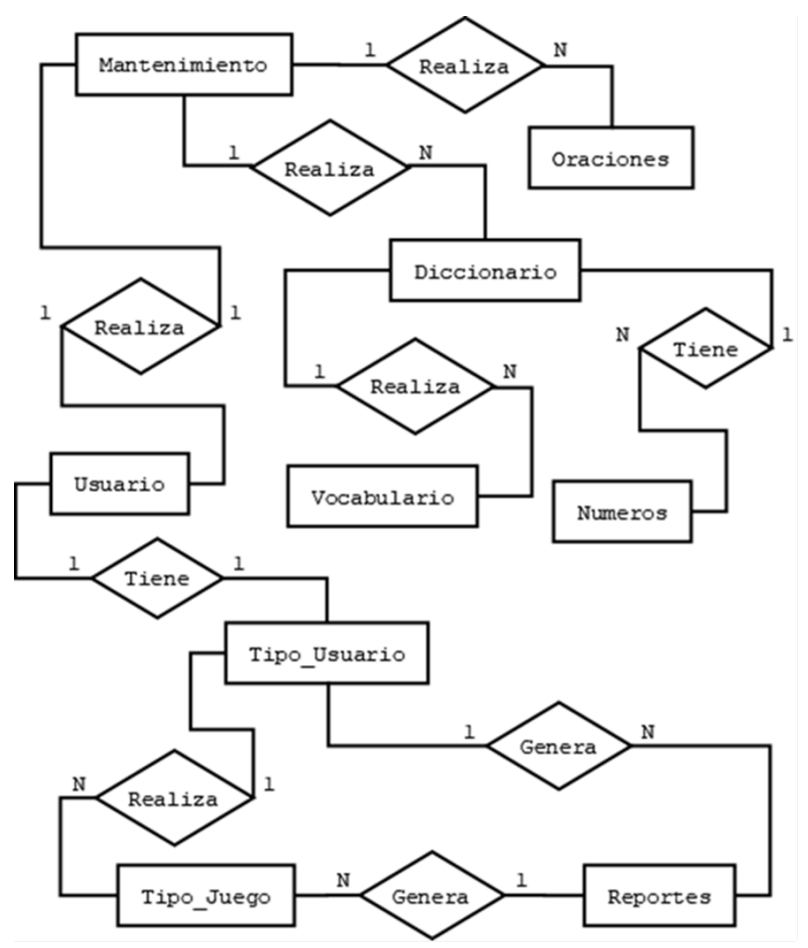

Una base de datos es un conjunto de tablas o entidades tiene una estrecha relación entre sí y contienen atributos con sus respectivos tipos de datos, en donde se almacenarán los datos manipulados en el sistema, como se puede ver en la Fig. 15:
Fig. 15. Base de Datos del Software de aprendizaje de la lengua de señas.

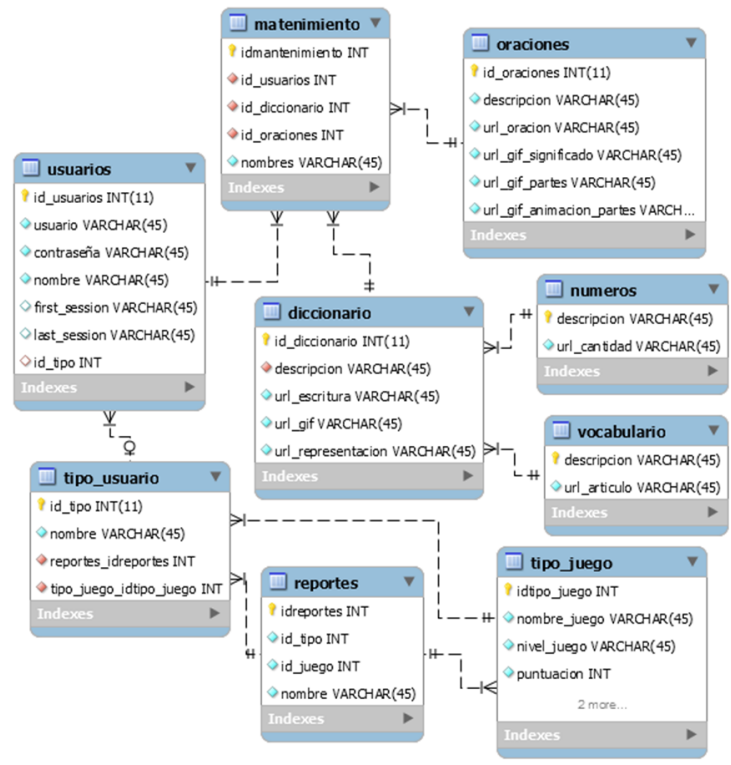

La Base de Datos del software de aprendizaje de la lengua de señas será alimentada por los usuarios administrador, docentes y estudiantes.

\section{Implementación y pruebas unitarias}

La construcción del software fue realizada por estudiantes de la carrera de Ingeniería de Sistemas de la Universidad Católica de Cuenca. Tomando en consideración, los resultados obtenidos del estudio preliminar y la parte instruccional de los estudiantes de la Unidad Educativa Especial Claudio Neira Garzón, se desarrolló el software utilizando el lenguaje de programación Java (NetBeans), MySQL (Base de Datos) y Flash e Illustrator (diseño gráfico).

El sistema cuenta con 2 tipos de usuarios:

- Administrador: es quien se encarga de la alimentación de información en las categorías contenidas en el programa (guardar, actualizar y eliminar).

- Usuario final: se divide en 2 tipos: profesores y estudiantes, son aquellos que harán uso de las distintas funciones del sistema.

El sistema permite al usuario la eseñanza-aprendizaje de la lectoescritura, tanto en el lenguaje español como en el lenguaje de señas, el mismo que está conformado por varios formularios, entre sus formularios principales están: diccionario, vocabulario y oraciones simples. Esta aplicación tiene una base de datos en la cual se almacena las imágenes, gifs e información de los diferentes formularios.

Las categorías y subcategorías fueron elaboradas en base al DICCI SEÑAS de la Pontificia Universidad Católica de Chile publicado en sus Tecnologías de Inclusión CEDETI http://diccisenas.cedeti.cl/AR/categoria/11/ y el Lenguaje de Señas Guía Básica sobre una Comunicación Especial - Tomo I de la página Sordos Ecuador http://www. sordosecuador.com/p/lenguaje-de-senas-guia-basica.html. 
La página principal del sistemas se puede ver en la Fig. 16:

Fig. 16. Pantalla inicial del sistema.

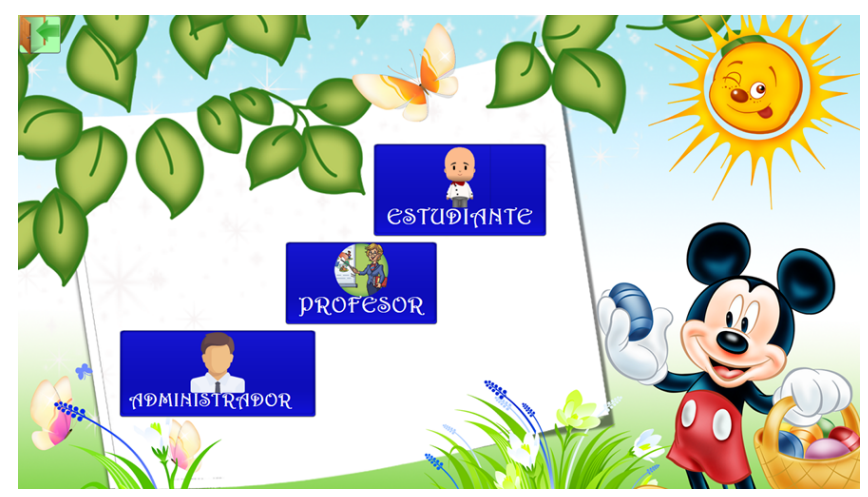

\section{Creación de oraciones simples}

Para la creación de una oración simple se utiliza el formulario oraciones simples, el mismo que existe tanto para docentes como estudiantes y se apoya en los formularios de diccionario y vocabulario en caso de no saber el significado de alguna palabra, así mismo, se tiene juegos que permiten reforzar el aprendizaje del diccionario, vocabulario y oraciones simples; también hay un formulario de reportes de estudiantes que permite dar un seguimiento del aprendizaje de los mismos.

La estructura gramatical del lenguaje de señas tiene una interpretación diferente al momento de ser traducida al lenguaje español, su estructura gramatical es la siguiente:

$$
\text { SUJETO + PREDICADO + VERBO }
$$

( $\mathrm{Si}$ la oración tiene tiempo, es decir está en pasado o en futuro seria de la siguiente forma)

TIEMPO + SUJETO + PREDICADO + VERBO

El sistema busca corregir las deficiencias gramaticales y mejorar el léxico del lenguaje español y optimizar el aprendizaje en los estudiantes de la lectoescritura a través del diccionario, vocabulario, juegos y oraciones simples.

Fig. 17. Juego que permite crear una oración simple.

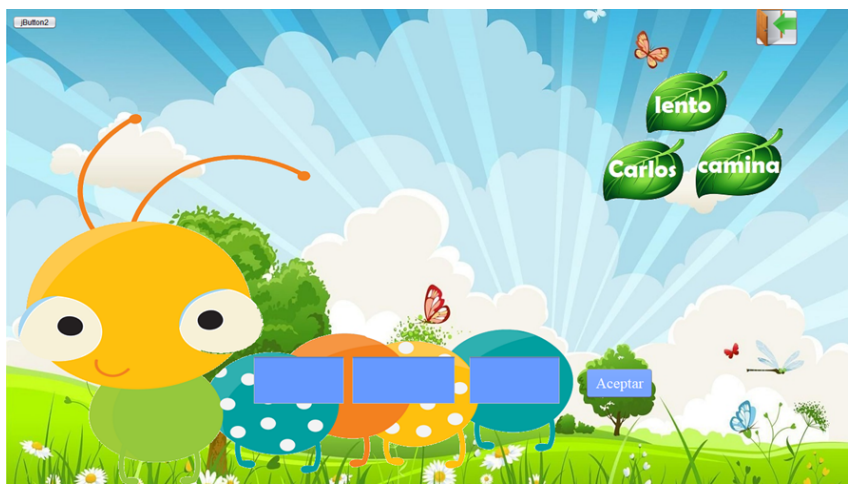

\section{Página del profesor}

El profesor a través de un micrófono puede introducir una palabra que es capturada mediante el Talking y compa- rada con su base datos, este palabra capturada es también comparada con un palabra almacenada en una base de datos MySQL que permite la visualización de la escritura, representación y el significado de la palabra en lenguaje de señas.

El profesor puede hacer uso del diccionario, vocabulario, oraciones simples y reportes de estudiantes:

- El diccionario tiene información de las subcategorías: alimentos y bebidas, acciones y cualidades, escuela, hogar, días y meses, ocupaciones y términos lingüísticos.

- El vocabulario tiene información de las categorías: alimentos y bebidas, escuela, hogar, días y meses y ocupaciones.

- Las oraciones simples tienen información de: alimentos y bebidas, acciones y cualidades, escuela, hogar y términos lingüísticos.

- Reporte de estudiantes: ayuda al profesor a obtener una visión del aprendizaje y rendimiento de sus estudiantes con respecto al aprendizaje de los contenidos de la asignatura a través del uso del programa, además, permite generar estadísticas de los tiempos que los estudiantes emplean para realizar cada actividad y retroalimentar su instrucción, gracias a las mismas se puede saber también, los contenidos que los estudiantes están con mayores dificultades en su aprendizaje.

Dentro de está página, el profesor enseña la lectoescritura del lenguaje español y el lenguaje de señas de una forma más entretenida y dinámica. Ya que en la misma, se muestra la Escritura (Lenguaje Español), la Representación (Imagen) y la Interpretación (Lengua de Señas), como se puede ver en la Fig. 18.

Fig. 18. Pantalla de presentación del vocabulario.

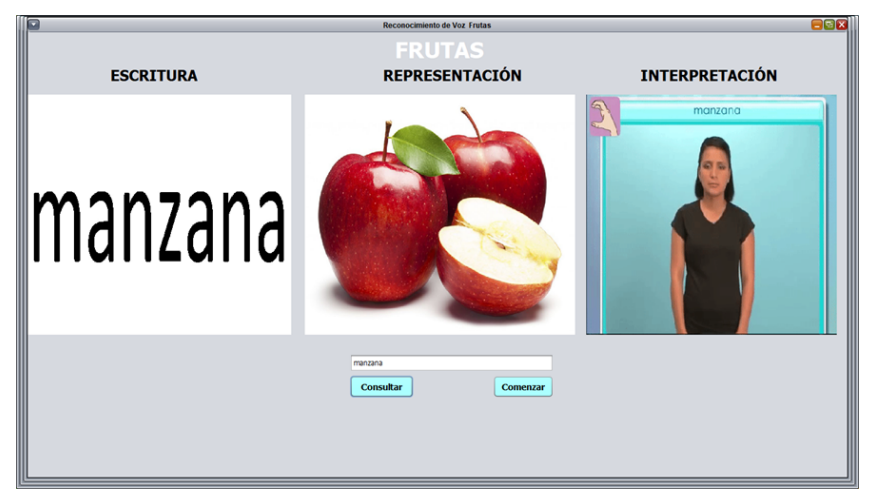

Además, los profesores en el caso que tengan una discapacidad auditiva podrán hacer uso del teclado para la utilización del sistema con todas sus funcionalidades.

\section{Página del estudiante}

El estudiante a través de esta página puede hacer uso de las siguientes opciones: diccionario, vocabulario y oraciones simples: 
- El diccionario tiene un juego de memoria que permite encontrar las palabras o imágenes pares.

- El vocabulario tiene un juego que permite unir un artículo con un sustantivo.

- Las oraciones simples tienen un juego que permite completar una oración.

Todos los juegos tienen 3 niveles de complejidad, existe un nivel básico, intermedio y avanzado; la información presentada en los juegos, son recuperados desde una base datos y mostrados de forma aleatoria en cada uno de los formularios. Los mismos, que permitirán reforzar el aprendizaje en los estudiantes.

\section{Integración y pruebas del sistema}

El sistema ha sido integrado de acuerdo a los requerimientos de los usuarios y probado de forma continua a través de secciones programadas con los estudiantes y los docentes, lo que ha permitido realizar cambios significativos y obtener un sistema muy amigable para con los estudiantes y docentes. Algunas de sus apreciaciones fueron:

- La interfaz gráfica de la aplicación es del agrado de los estudiantes, docentes y muy intuitiva en su manipulación.

- El uso de los juegos no presenta dificultad en los estudiantes, lo pudieron realizar con indicaciones cortas.

- Su uso en el aula permite complementar los aprendizajes de los estudiantes y las explicaciones del profesor.

- La herramienta fomenta el desarrollo de habilidades de pensamiento (crear, analizar y evaluar).

- Al realizar las pruebas a un niño con discapacidad auditiva se notó que mejoró su habilidad de memoria y gramática del lenguaje español reduciendo sus tiempos en los juegos y ordenando de mejor manera las oraciones.

Sin embargo, el sistema esta en su primera etapa de desarrollo, al mismo que se seguirá alimentando mas funcionalidades que le permita abordar los contenidos en su totalidad de la asignatura de Lengua y Literatura.

\section{E. Operación y mantenimiento}

Se ha registrado información del diccionario, vocabulario, oraciones simples y juegos:

- En el diccionario se registró información de las subcategorías: alimentos y bebidas, acciones y cualidades, escuela, hogar, días y meses, ocupaciones y términos lingüísticos.

- En el vocabulario se registró información de las categorías: alimentos y bebidas, escuela, hogar, días y meses y ocupaciones.

- En las oraciones simples se registró información de: alimentos y bebidas, acciones y cualidades, escuela, hogar y términos linguiísticos. Además, las oraciones simples se complementa con un juego que permite completar una oración.
La operación y mantenimiento del Software de aprendizaje de la lengua de señas es un proceso continuo que se realiza de acuerdo a los contenidos de la asignatura de Lengua y Literatura, material didáctico y las necesidades educativas de los profesores y estudiantes. Además se pueden crear nuevas categoría y subcategorías.

\section{DISCUSIÓN}

La evaluación del sistema con los estudiantes, a través de una ficha de observación, de la facilidad de uso del software y su utilidad en el proceso de enseñanza-aprendizaje de la lectoesritura, arrojo los resultados que se puede ver en la Fig. 19:

Fig. 19. Resultados de la ficha de observación aplicada a los estudiantes en el momento de utilizar el sistema.

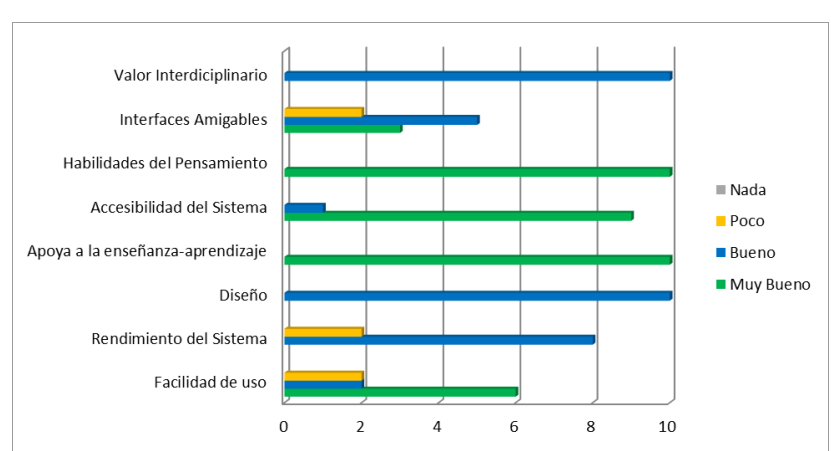

Esto determina que el $47 \%$ del sistema es Muy Bueno e interactivo de acuerdo a la ficha de observación aplicada a los estudiantes en el momento de utilizar el sistema, asimismo el $45 \%$ es Bueno, el $8 \%$ Poco y el $0 \%$ Nada. Haciendo un análisis se interpreta que el sistema contribuye con las habilidades del pensamiento (crear, analizar y evaluar), apoya el proceso de enseñanza-aprendizaje, es accesible para niños con discapacidad auditiva (traduce de voz a lengua de señas), es fácil de usar y sus interfaces son amigables.

\section{CONCLUSIONES}

Se concluye que el sistema fue desarrollado en su primera etapa, el mismo que apoya el proceso de enseñanzaaprendizaje de los niños con discapacidad auditiva en la asignatura de Lengua y Literatura de la Unidad Educativa Claudio Neira Garzón dentro de la lectoescritura, los formularios diseñados en el sistema son: Profesor, Estudiante y Administrador. Los formularios del Profesor y Estudiante tienen formularios de diccionario, vocabulario, oraciones simples y juegos. Los juegos tienen tres niveles de complejidad y el formulario del Profesor además tiene el reporte de estudiantes.

En las pruebas realizadas con el sistema se han obtenido muy buenos resultados, teniendo una excelente acogida por parte de los docentes y estudiantes que demostraron mucho interés por usar el software para la enseñanza-aprendizaje, en el análisis del uso del programa de los niños arrojo 
buenos resultados y también sugerencias de cambios en el mismo para mejorar su interactividad con los estudiantes y profesores. Además, a través del uso del sistema los niños incrementaron el nivel de compresión y entendimiento del lenguaje español y la lengua de señas.

Finalmente, el software que con el uso de un dispositivo de reconocimiento de voz traduce a lenguaje de señas sirve como herramienta de trabajo en la labor educativa de la Unidad Educativa Especial Claudio Neira Garzón en la asignatura Lengua y Literatura, permitiendo la creación de oraciones simples de forma interactiva y dinámica. Este software esta orientado principalmente a niños entre 7 y 15 años con déficit auditivo parcial o total, además, beneficia a personas adultas y familiares de las personas con déficit auditivo parcial o total, entre otras personas.

\section{REFERENCIAS}

[1] T. Constitucional, "Constitución de la república del ecuador," Quito-Ecuador: Registro Oficial, vol. 449, pp. 20-10, 2008.

[2] S. N. de Planificación y Desarrollo, "Plan nacional de desarrollo 2017-2021. toda una vida.," Ecuador, Septiembre 2017.

[3] CONADIS, "Normas jurídicas en discapacidad ecuador.," Mayo 2014.

[4] B. MUNDIAL, "Informe mundial la discapacidad," Educación, vol. 218, no. 219, p. 219, 2011.

[5] CONADIS, "Información estadística de personas con discapacidad.," Marzo 2018.

[6] M. A. C. Molina, J. K. V. Durán, and D. A. R. Espinosa, "Accesibilidad web en las instituciones de salud de la ciudad de cuenca. análisis preliminar," in $I V$ Conferencia Internacional sobre Aplicación de Tecnologías de la Información y Comunicaciones para mejorar la Accesibilidad (ATICAcces 2016), pp. 125132, Servicio de Publicaciones, 2016.

[7] J. K. V. Durán, M. A. C. Molina, and D. X. P. Japón, "Funciones de accesibilidad que logran y mejoran las competencias de uso y manejo de los teléfonos inteligentes," in CIIEE 2017, Marzo 2017.

[8] M. A. C. Molina, J. K. V. Durán, and V. S. E. Jara, "Accesibilidad web en las instituciones de salud de la ciudad de cuenca. análisis preliminar," in VIII Congreso Internacional sobre Aplicación de Tecnologías de la Información y Comunicaciones Avanzadas (ATICA2017), V Conferencia Internacional sobre Aplicación de Tecnologías de la Información y Comunicaciones para mejorar la Accesibilidad, I Congreso Internacional Educar en la Sociedad Red: Realidad, Retos y Perspectivas, pp. 141-148, Servicio de Publicaciones, 2017.

[9] CONADIS, “Causas de discapacidad,” Mayo 2013.

[10] M. Figueroa Cruz, G. Vázquez Zubizarreta, and M. A. Campoverde Molina, "Software educativo para el desarrollo de habilidades de la conducta adaptativa en personas con discapacidad intelectual," VARONA ISSN: 1992-8238, no. 61, p. 11, 2015.

[11] R. Claros-Kartchner, "La inclusión de las personas sordas, como grupo étnico, en los sistemas educativos," 2017.

[12] A. Ecuador, "Ley orgánica de discapacidades," Registro Oficial N, vol. 796, Septiembre 2012.

[13] M. P. Godoy, S. González, and M. Verdugo, "Guía de apoyo técnico-pedagógico: Necesidades educativas especiales en el nivel de educación parvularia," Santiago de Chile: Editorial Atenas, p. 46, 2008.

[14] S. Cano, J. Muí, C. Collazos, V. B. Amador, et al., "Aplicación móvil para el aprendizaje de la lectoescritura con fitzgerald para niños con discapacidad auditiva," in Anais dos Workshops do Congresso Brasileiro de Informática na Educação, vol. 4, p. 240, 2015.

[15] X. C. Alejandro Oviedo and R. Cabezas, "Ecuador, atlas sordo," 2015.

[16] C. Becerra Sepúlveda, "Lenguaje y educación en niños sordos: encuentros y desencuentros," REXE. Revista de Estudios y Experiencias en Educación, no. 14, 2008.

[17] D. L. Parra and M. Luque-Rojas, "Necesidades específicas de apoyo educativo del alumnado con discapacidades sensorial y motora," Summa psicológica UST (En línea), vol. 10, no. 2, pp. 57-72, 2013.

[18] E. R. Linares, "El aprendizaje de la lectoescritura en los niños y niñas sordos," Caleidoscopio, Revista digital de contenidos educativos, no. 2, p. 1, 2009.

[19] S. E. A., "El podcasts en la enseñanza de la lectoescritura," Revista Virtual Universidad Católica del Norte, pp. 9-26, 2012.

[20] R. Montealegre and L. A. Forero, "Desarrollo de la lectoescritura: adquisición y dominio," Acta colombiana de psicología, vol. 9, no. 1, pp. 25-40, 2006.

[21] M. Gértrudix, S. Álvarez, A. Galisteo, M. del Carmen Gálvez, and F. Gértrudix, "Acciones de diseño y desarrollo de objetos educativos digitales: programas institucionales," RUSC. Universities and Knowledge Society Journal, vol. 4, no. 1, 2007.

[22] M. de Educación, "Circular nro. mineduc-ve-201600001-c," p. 12, Agosto 2016.

[23] S. B. Stainback, "L'educació inclusiva: definició, context i motius," Suports: revista catalana d'educació especial i atenció a la diversitat, vol. 5, no. 1, pp. 1825, 2001.

[24] "Educación inclusiva," 2006.

[25] G. Echeita Sarrionandia and C. Duk Homad, "Inclusión educativa," REICE. Revista Iberoamericana sobre Calidad, Eficacia y Cambio en Educación, vol. 6, no. 2, 2008.

[26] M. Granada Azcárraga, M. P. Pomés Correa, and S. Sanhueza Henríquez, "Actitud de los profesores hacia la inclusión educativa," Papeles de trabajo-Centro de Estudios Interdisciplinarios en Etnolingüística y Antropología Socio-Cultural, no. 25, pp. 0-0, 2013. 
[27] V. Esteller and E. Medina, "Procesos de desarrollo de software y materiales educativos computarizados," Eduweb, Revista de Tecnología de Información y Comunicación en Educación, vol. 6, no. 1, 2012.

Aceptado: 15 de junio de 2018

[28] X. F. Grau and M. I. S. Segura, "Desarrollo orientado a objetos con uml," Recuperado el, vol. 1, 2008.

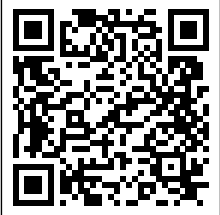

